Annuaire suisse de politique de développement

27-2 | 2008

Migration et développement: un mariage arrangé

\title{
Eclairage - Transferts de fonds : enquête auprès des immigrés d'origine serbe en Suisse
}

Mathias Lerch

\section{OpenEdition}

1 Journals

Édition électronique

URL : http://journals.openedition.org/aspd/509

DOI : 10.4000/aspd.509

ISSN : 1663-9669

Éditeur

Institut de hautes études internationales et du développement

Édition imprimée

Date de publication : 1 décembre 2008

Pagination : 161-166

ISBN : 978-2-940415-07-6

ISSN : 1660-5934

Référence électronique

Mathias Lerch, «Eclairage - Transferts de fonds : enquête auprès des immigrés d'origine serbe en Suisse ", Annuaire suisse de politique de développement [En ligne], 27-2 | 2008, mis en ligne le 22 mars 2010, consulté le 08 septembre 2020. URL : http://journals.openedition.org/aspd/509 ; DOI : https:// doi.org/10.4000/aspd.509

(c) The Graduate Institute I Geneva 


\title{
Eclairage
}

\section{Transferts de fonds: enquête auprès des immigrés d'origine serbe en Suisse}

\author{
Mathias Lerch*
}

L a migration constitue un potentiel pour le développement des pays d'origine et son impact sur l'allègement de la pauvreté n'est plus discuté. Les montants d'argent transférés par les migrants (remittances) peuvent parfois même augmenter dans des contextes de crises politiques ou économiques, comme lors de la transition qui caractérise la Serbie depuis 1992². Ces "transferts personnels", tels que définis dans le cadre de la balance des paiements ${ }^{3}$, constituent un important flux financier international et dépassent le montant de l'aide publique au développement. Connaître le comportement économique des migrants envers leur pays d'origine et identifier les déterminants de leurs pratiques revêt ainsi une importance certaine, notamment pour I'harmonisation et la cohérence des politiques migratoires et de coopération au développement.

A cet égard, les enquêtes populationnelles auprès des migrants fournissent un outil de mesure flexible. D'une part, elles permettent d'améliorer la qualité des estimations macroéconomiques des flux de remittances, encore lacunaires jusqu'à nos jours. L'importance des différents flux bilatéraux émanant d'un pays d'immigration - soit des corridors de remittances - peut ainsi être établie. D'autre part, les enquêtes fournissent des informations qualitatives sur les pratiques d'envois de fonds des migrants et sur leur niveau d'intégration dans le pays d'accueil ${ }^{4}$. Ces informations sont susceptibles d'orienter les politiques de coopération au développement afin de tirer profit de la complémentarité entre les aides privées et publiques.

Cette contribution expose les principaux résultats de la première enquête relative aux pratiques de transferts de fonds des migrants établis en Suisse. Elle se réfère spécifiquement aux migrants d'origine serbe, c'est-à-dire les serbophones, soit un sousgroupe au sein de la population de nationalité serbo-monténégrine telle que définie par

* Collaborateur scientifique au Laboratoire de démographie et d'études familiales, Faculté des sciences économiques et sociales, Université de Genève.

1 R.H. Adams and J. Page, "Do international Migration and Remittances Reduce Poverty in Developing Countries?», World Development, vol. 33, n 10, 2005, pp. 1645-1669.

2 M. Schrooten, Bringing Home the Money: What Determines Workers' Remittances to Transition Countries, Discussion Paper Series A, $n^{0}$ 466, Institute of Economic Research (IER), Tokyo, Hitotsubashi University, 2005.

3 II s'agit de tous les transferts monétaires ou de biens effectués par des ménages résidents vers des ménages situés dans le pays d'origine durant les douze mois précédant l'enquête dont rend compte la présente contribution. Voir: A. Alfieri and I. Havinga, Issue Paper: Definition of Remittances, Meeting of the United Nations Technical Subgroup on Movement of Persons - Mode 4, New York, United Nations, 22-24 February 2006.

4 M. Lerch, P. Wanner and W. Haug, The Measurement of Remittances in Industrialized Countries - or How to Limit Multiple Biases, Background Paper for the Joint UNECE/World Bank/US Census Bureau Expert Group Meeting on the Contribution of Household Surveys to Measuring Remittances, Suitland (United States), 14-15 January 2008. 
la statistique suisse en 2006. L'importante partie albanophone (en majorité des Kosovars) ou d'origine monténégrine de cette population n'est pas couverte par l'enquête ${ }^{5}$. II est par conséquent impossible d'identifier précisément la population étudiée par le biais des statistiques officielles. Ces dernières relèvent toutefois la dépendance importante du pays d'origine dans son ensemble envers les remittances: ceux-ci ont en effet atteint 4129 millions de dollars en 2005, ce qui fait de ce pays le premier bénéficiaire dans les Balkans et le situe parmi les dix principaux pays bénéficiaires au monde (en pourcentage du PIB) 6 .

\section{La spécificité de la migration serbe et l'enquête auprès des migrants en Suisse}

La migration d'origine serbe en Suisse remonte au début du $x x^{e}$ siècle, à la suite d'une convention consulaire avec le royaume de Serbie facilitant l'immigration dans le cadre des relations économiques, politiques et culturelles entre les deux pays? ${ }^{7}$. Le gouvernement yougoslave institué en 1945 a dès 1967 activement promu des migrations temporaires dans un contexte de pression démographique et économique ${ }^{8}$. Cependant, la mise en place par la Suisse du modèle d'admission des trois cercles en 1990, qui excluait l'ancienne Yougoslavie des bassins de recrutement, a rendu difficile la migration économique en provenance de ce pays? .

La pression à l'émigration est toutefois restée élevée en raison des guerres civiles et de la transition économique. La population de nationalité serbe établie en Suisse s'est fortement accrue durant les années 1980 et 1990 pour atteindre 187365 individus à la fin 2007, Kosovars inclus ${ }^{10}$; elle représente désormais $12 \%$ des étrangers contre seulement 2,3\% en 1970. Cet accroissement s'explique par l'évolution des motifs de migration durant les années 1990, davantage humanitaires ou liés au regroupement familial qu'auparavant ${ }^{11}$.

La population d'origine serbe en Suisse se distingue ainsi des autres ressortissants de I'ancienne Yougoslavie (comme les Kosovars ou les Macédoniens) par sa stabilité, sa forte composante familiale et son niveau de formation et d'intégration économique proportionnellement plus élevér ${ }^{2}$. L'impact économique des migrants est donc potentiellement le plus important dans l'actuelle Serbie.

Le Secrétariat d'Etat à l'économie (SECO) a, pour cette raison, lancé une recherche-action sur le corridor des remittances établi entre la Suisse et l'actuelle Serbie, avec pour objectif

5 Au moment de l'enquête, la population de nationalité serbo-monténégrine était composée d'individus d'origine serbe, monténégrine ou kosovare et d'autres minorités ethniques. Ces trois groupes principaux constituent aujourd'hui des Etats indépendants. Nous utiliserons par conséquent la dénomination "d'origine serbe" pour désigner le groupe ethnique sur lequel a porté l'enquête.

6 M. Schrooten, op. cit.

7 D. Mikic, "Historische Hintergründe zur Migration in die Schweiz», in Einblicke: Serbinnen und Serben in der Migration, Fachstelle für interkulturelle Fragen der Stadt Zürich, November 2001.

8 I.G. Molnar, "The Sociology of Migration from the Former Yugoslavia», New Community, vol. 23, n 1, 1997, pp. 109-122.

9 D. Efionayi, J.-M. Niederberger and P. Wanner, "Switzerland Faces Common European Challenges», Migration Information Souce (MPI), Washington, DC, Migration Policy Institute, 2005, <http://www.migrationinformation. org/Feature/print.cfm?ID=284> (consultation: 10 janvier 2008).

10 Source: Office fédéral des migrations (ODM), <http://www.bfm.admin.ch/bfm/fr/home/themen.html> >statistiques $>$ statistique des étrangers >résultats actuels (consultation: 2 juin 2008).

11 D.M. Gross, Immigration to Switzerland: The Case of the Former Republic of Yugoslavia, World Bank Policy Research Working Paper, $n^{0}$ 3880, Washington, DC, The World Bank, 2006.

12 M. Lerch, J. Dahinden and P. Wanner, Mapping Three Balkan Diasporas in Switzerland, Neuchâtel, Swiss Forum for Migration and Population Studies, 2005 (unpublished work). 
l'étude des pratiques de transferts, de l'efficience du système bancaire et de l'utilisation de ces fonds dans le pays d'origine. Les informations ont été récoltées lors d'une enquête téléphonique auprès de 600 ménages d'origine serbe en mars 2006. Le Registre central des étrangers, qui fournit la liste exhaustive des noms et adresses des ressortissants de la Serbie-et-Monténégro, a constitué la base de l'échantillonnage des ménages d'origine serbe. Les résultats ne peuvent cependant pas être généralisés à l'ensemble de cette communauté puisque la définition précise de la base d'échantillonnage - soit la population de référence de cet échantillon - s'est avérée difficile en raison de l'absence d'information sur l'appartenance ethnique des individus ${ }^{13}$.

\section{L'importance et les déterminants de l'envoi de fonds des migrants serbes en Suisse}

Les analyses de l'enquête ont montré qu'une large majorité des migrants d'origine serbe établis en Suisse entretiennent des relations économiques avec leur pays d'origine: trois quarts des ménages interrogés ont envoyé de l'argent ou des biens. Le montant médian transféré s'élève à 3000 francs par an et par ménage vivant en Suisse et se répartit entre plusieurs destinataires, composés principalement des grands-parents (45\%), de la fratrie $(20 \%)$ ou des membres de la propre famille laissée au pays d'origine (11\%).

Ces transferts représentent un apport financier important à l'économie ménagère des bénéficiaires: le montant médian perçu en Serbie se situe à 1000 francs par an pour chaque destinataire, ce qui représente $20 \%$ de plus que le seuil de pauvreté extrême et la moitié du seuil absolu ${ }^{14}$. En effet, quatre ménages sur cinq pratiquant ces envois transfèrent de l'argent dans le but de soutenir la consommation de biens courants ou les dépenses dans le domaine de la santé.

La propension importante de la communauté d'origine serbe à l'envoi de fonds est due à plusieurs facteurs. D'une part, elle n'est pas affectée par la durée de séjour des immigrés, quand bien même les montants versés diminuent avec les années vécues en Suisse. Cela contraste avec ce qui se produit dans la majorité des pays d'immigration, où la propension à I'envoi décline avec l'accroissement de la durée de séjour. La dégradation du niveau de vie durant les années 1990 consécutive aux conflits armés et à la transition économique a probablement (re)mobilisé le soutien économique des migrants serbes au pays d'origine. D'autre part, la proximité géographique entre le pays d'origine et le pays d'accueil facilite le maintien des relations sociales et économiques transnationales. Celles-ci constituent d'importants déterminants des fonds transférés par les Serbes: les migrants qui ont maintenu des réseaux en Serbie, qui y voyagent souvent et qui y possèdent un compte bancaire ont non seulement une probabilité accrue de transférer de l'argent, mais envoient aussi des sommes plus conséquentes.

Cependant, les conditions de vie des migrants en Suisse sont susceptibles de limiter les capacités de soutien financier au pays d'origine. En particulier, la position dans le cycle de vie familiale et le niveau d'intégration économique déterminent les pratiques des transferts de fonds serbes. Contrairement à d'autres contextes migratoires dans le monde, les ménages aisés d'origine serbe en Suisse ont davantage tendance à envoyer de l'argent. Or, les montants effectivement transférés augmentent dans l'absolu avec l'accroissement du niveau de formation et

13 Voir: M. Lerch, J. Dahinden and P. Wanner, Remittance Behaviour of Serbian Migrants in Switzerland, SFM Studies, $n^{0}$ 51, Neuchâtel, Swiss Forum for Migration and Population Studies, 2007.

14 World Bank, Serbia and Montenegro: Poverty Assessment, Report $n^{\circ}$ 26011-YU, vol. 2, Poverty Reduction and Economic Management Unit, Europe and Central Asia Region, Washington, DC, The World Bank, 2003. 
I'amélioration de la situation économique du ménage, à l'instar de ce qu'on observe dans la plupart des pays d'immigration. Les migrants récemment naturalisés envoient aussi plus d'argent, bien que moins fréquemment. Ces caractéristiques sociodémographiques sont décisives pour une intégration réussie dans le marché du travail. Or, les charges économiques des jeunes familles immigrées grèvent souvent le budget disponible pour le soutien au pays d'origine. En effet, les ménages non familiaux ou chez qui les enfants sont adultes et vivent encore dans le ménage parental ont une probabilité plus élevée de transférer de l'argent.

En dernier lieu, I'utilisation des canaux bancaires est d'une importance particulière pour le processus de développement économique du pays d'origine. Les remittances transitant par ce biais dans le pays soutiennent l'intermédiation financière des banques, et donc l'offre de crédits d'investissement. Or, moins d'un cinquième des ménages serbes qui envoient des fonds utilisent des canaux formels - le plus souvent bancaires. La majorité des envois sont effectués de main à la main. Non seulement ce procédé est plus rapide, mais il est aussi considéré comme plus sûr comparé aux virements bancaires. II est probable que le gel des comptes des émigrés sous l'ère Milosevic et la faillite des systèmes bancaires pyramidaux durant les années $1990^{15}$ pèsent encore aujourd'hui sur le niveau de confiance accordé au système bancaire local. Le marché bancaire s'est cependant stabilisé et l'arrivée des banques étrangères s'est fortement accrue depuis la fin de la guerre au Kosovo.

La faible utilisation des canaux formels s'explique en partie par un manque d'information: les personnes âgées ou maîtrisant peu la langue parlée dans le lieu de vie utilisent le moins ces canaux. Les Serbes dont le réseau amical est davantage centré sur la Suisse transfèrent en outre plus souvent leur argent par les banques. La connaissance du système bancaire actuel et de ses avantages s'accroît sans doute avec le processus d'intégration. Bien que le coût d'un virement bancaire soit inférieur aux tarifs pratiqués par les agences spécialisés, le système bancaire manque toutefois de transparence: les tarifs supplémentaires imposés par les banques destinataires dans le pays d'origine sont souvent inconnus des banques suisses.

\section{Conclusion}

Jadis le pays le plus riche de la région, la Serbie a connu durant les années 1990 une chute considérable du niveau de vie à la suite des conflits ainsi que de la transition économique, ce qui a (re)mobilisé en masse le soutien de la communauté émigrée. Les transferts de fonds des migrants d'origine serbe établis en Suisse allègent la pauvreté, particulièrement au sein de la population âgée, mais produisent peu d'investissements au niveau du ménage.

Les résultats de l'enquête ont également montré le rôle potentiel des politiques des pays d'accueil par rapport à l'ampleur et à l'impact des transferts de fonds. D'abord, ils soulignent l'importance des relations transnationales des migrants, dont les remittances font partie intégrante ${ }^{16}$. La mise en place de conditions favorisant le maintien des liens avec le pays d'origine - comme par exemple la libre circulation des résidents immigrés ou le vote serbe à l'étranger pourrait donc accroître ces flux financiers. Ensuite, les chances d'intégration économique offertes aux migrants déterminent le budget disponible pour le soutien au pays

15 Bank Austria, Banking in Serbia and Montenegro, Extra Report, Economics Department, Bank Austria Creditanstalt AG, 2005. World Bank, op. cit.

16 L.E. Guarnizo, "The Economics of Transnational Living», International Migration Review, vol. 37, n 3, 2003, pp. 666-699. 
d'origine. Enfin, le regroupement familial freine les envois et diminue les sommes transférées, les ressources étant d'abord allouées à l'éducation des jeunes enfants. Des politiques axées sur la migration individuelle de travail augmenteraient sans doute les transferts. En outre, des interventions politiques sur les conditions financières des envois sont également envisageables. La diffusion d'informations relatives aux canaux de transferts formels ainsi qu'à leurs avantages $^{17}$ serait à même d'accroître leur utilisation. L'intermédiation financière des banques dans le pays d'origine et par conséquent l'offre de crédits d'investissement s'en trouveraient ainsi élargies.
Les enquêtes populationnelles ne fournissent cependant pas seulement des informations qualitatives. Elles peuvent aussi aider à compléter les estimations encore lacunaires des flux bilatéraux de remittances émanant de la Suisse. L'étude des comportements économiques des migrants envers leur pays d'origine par le biais d'enquêtes est donc susceptible de soutenir une intégration cohérente de la politique migratoire et de la politique de coopération au développement. En effet, les caractéristiques des (ménages) migrants sont, comparées aux facteurs macroéconomiques, les principaux déterminants des remittances ${ }^{18}$ et par conséquent de l'impact de la migration sur les pays d'origine.

\section{Bibliographie}

Adams, R.H. and J. Page, "Do international Migration and Remittances Reduce Poverty in Developing Countries?», World Development, vol. 33, n²10, 2005, pp. 1645-1669.

Alfieri, A. and I. Havinga, Issue Paper: Definition of Remittances, Meeting of the United Nations Technical Subgroup on Movement of Persons - Mode 4, New York, United Nations, 22-24 February 2006.

Bank Austria, Banking in Serbia and Montenegro, Extra Report, Economics Department, Bank Austria Creditanstalt $A G, 2005$.

Efionayi, D., J.M. Niederberger and P. Wanner, "Switzerland Faces Common European Challenges», Migration Information Souce (MPI), Washington, DC, Migration Policy Institute, 2005, <http://www.migrationinformation. org/Feature/print.cfm?ID=284> (consultation: 10 janvier 2008).

Gross, D.M., Immigration to Switzerland: The Case of the Former Republic of Yugoslavia, World Bank Policy Research Working Paper, $n^{0}$ 3880, Washington, DC, The World Bank, 2006.

Guarnizo, L.E., "The Economics of Transnational Living», International Migration Review, vol. 37, n 3, 2003 , pp. 666-699.

Lerch, M., J. Dahinden and P. Wanner, Mapping Three Balkan Diasporas in Switzerland, Neuchâtel, Swiss Forum for Migration and Population Studies, 2005 (unpublished work).

Lerch, M., J. Dahinden and P. Wanner, Remittance Behaviour of Serbian Migrants in Switzerland, SFM Studies, $\mathrm{n}^{\circ} 51$, Neuchâtel, Swiss Forum for Migration and Population Studies, 2007.

Lerch, M., P. Wanner and W. Haug, The Measurement of Remittances in Industrialized Countries - or How to Limit Multiple Biases, Background Paper for the Joint UNECE/World Bank/US Census Bureau Expert Group Meeting on the Contribution of Household Surveys to Measuring Remittances, Suitland (United States), 14-15 January 2008.

Mikic, D., "Historische Hintergründe zur Migration in die Schweiz», in Einblicke: Serbinnen und Serben in der Migration, Fachstelle für interkulturelle Fragen der Stadt Zürich, November 2001.

Molnar, I.G., "The Sociology of Migration from the Former Yugoslavia», New Community, vol. 23, n 1, 1997, pp. 109122.

Russel, S.S., "Remittances from International Migration: A Review in Perspective», World Development, vol. 14, $n^{\circ} 6,1986$, pp. 677-696.

17 Comme le pratique par exemple le gouvernement britannique.

18 S.S. Russel, "Remittances from International Migration: A Review in Perspective», World Development, vol. 14, $n^{0} 6,1986$, pp. 677-696. 
Schrooten, M., Bringing Home the Money: What Determines Workers' Remittances to Transition Countries, Discussion Paper Series A, n⿳0 466, Institute of Economic Research (IER), Tokyo, Hitotsubashi University, 2005.

World Bank, Serbia and Montenegro: Poverty Assessment, Report n 26011-YU, vol. 2, Poverty Reduction and Economic Management Unit, Europe and Central Asia Region, Washington, DC, The World Bank, 2003. 